\title{
A NOTE ON WIRTINGER-BEESACK'S INTEGRAL INEQUALITIES
}

\author{
Sin-EI TAKAHASI AND TAKESHI MIURA
}

Abstract. In this note we show that Wirtinger's integral inequalities and Beesack's integral inequality follow from a unified integral inequality. We also consider Banach space valued versions of these inequalities.

Mathematics subject classification (2000): 26D15.

Key words and phrases: Wirtinger's integral inequality; Beesack's integral inequality.

\section{REFERENCES}

[1] J. B. Diaz and F. T. Metcalf, Variations of Wirtinger's inequality, 1967 Inequalities (Proc. Sympos. Wright-Patterson Air Force Base, Ohio, 1965), 79-103, Academic Press, New York.

[2] B. FLORKIEWICZ AND K. WOJTECZEK, On some further Wirtinger-Beesack integral inequalities, Demonstratio Math. 32 (1999), 495-502.

[3] K. OZEKI AND N. OZEKI, Invitation to Inequalities, Kindai Kagakusya, 1987 (Japanese). 